\title{
A Brief Introduction to Natural Language Generation within Computational Creativity
}

\author{
Ben Burtenshaw \\ Computational Linguistics \& Psycholinguistics Research Center, and \\ The Antwerp Centre for Digital Humanities and Literary Criticism \\ The University of Antwerp, Prinsstraat 13, Antwerp, Belgium \\ benjamin.burtenshaw@uantwerpen.be
}

\begin{abstract}
This paper is an introduction to The 3rd Workshop on Computational Creativity in Language Generation (CC-NLG 2018), collocated with The 11th International Conference on Natural Language Generation (INLG 2018). The workshop acts as an overview of projects from the Computational Creativity field present within the general field of Natural Language Generation, and references further reviews within the area. Accepted papers to the workshop will use contemporary NLG methods or approach NLG problems from a creative perspective. The workshop intends to showcase creative applications as a worthwhile and meaningful pursuit to other NLG researchers.
\end{abstract}

\section{Introduction}

This short paper will introduce the workshop Computational Creativity in Natural Language Generation (CCNLG), and point to a limited portion of the field that surrounds it. CCNLG is intended as a discussion of recent advancements within Computational Creativity (CC) that are relevant to the field of Natural Language Generation (NLG), or utilize NLG methods. Collocated with The International Conference on Natural Language Generation (INLG), it also attempts to showcase how creative applications can utilise innovative NLG methods toward novel applications. This paper acts as brief introduction for researchers from outside the $\mathrm{CC}$ field.

Language has sat at the core of $\mathrm{CC}$ research since the field's origins. NLG projects by Meehan, Gervas, Colton, and Veale are key examples of $\mathrm{CC}$, as well as methodological cornerstones to researchers finding their way in a theo- retically nuanced arena. In turn, language generation projects have become key reference points to $\mathrm{CC}$ researchers working in other application areas. In short, Computational Creativity within Natural Language Generation is by no means an underdeveloped research trajectory; however, research comes from a broad range of venues and events, and there is a lack of discussion for concerns specific to the field itself. Conferences that have hosted CCNLG research include, INLG, The conferences of the Association for Computational Linguistics ( $\mathrm{ACl}, \mathrm{nAC}-\mathrm{ACL}, \mathrm{EACL})$, the International Conference on Computational Creativity (ICCC), as well as its predecessor workshops at ECAI, IJCAI, ECCBR and ICCBR, various AISB symposia on creativity and poetry, Computational models of Narrative workshop, International Conference on Interactive Digital Storytelling, the Intelligent Narrative Technologies workshop series. In recent years NLG has become relevant to the general public through creative application in dialogues systems, chatbots, and twitter bots. These widely available tasks have thrown creative language generation into the public eye, opening up a broad base of possible users. Typically though, NLG has been used by CC researchers in three formal areas; story generation, poetry generation, and joke generation. That said, CC researchers have used NLG in many applications from recipe to lyric generation. As well as introducing application within CC in NLG, this paper will also reference relevant literature reviews that support the workshop.

\section{Story Generation}

Stories are a fundamental part of creative writing, and have been generated by $\mathrm{CC}$ researchers for decades [3]. Furthermore, narratives are a crucial part of language outside of formal stories, and re- 
searchers have used NLG to produce informal stories [4].

An early example of creative story generation is TALE-SPIN, which generated Aesop's Fables based on the user's input (Meehan, 1977). The user was asked a series a questions during creation, that TALE-SPIN combined with prior knowledge on story attributes to generate a tale. The user could also chose from a set of morals, like "never trust flatterers", which the system would then attempt to communicate through the story. In essence, TALE-SPIN was a goal based problem solving system, that saw the attributes of the proposed Aesop fable as a problem, that it could solve via the inclusion of the correct knowledge pieces in a story. Also using a knowledge based approach, Universe by Michaeal Lebowitz draws on a database of character definitions and plot outlines to create stories around a user proposed theme [10]. McIntyre and Lapata developed one of the first search-based narrative systems that used user-input to search a database for relating phrases [12]. Gervas 2009 gives a detailed overview of story generation advancements from 1974 to 2009 [8].

\section{Poetry Generation}

Poems are an important part of how humans use language to be creative. Outside of formal poetry, from metaphor to analogy, poetic language litters our public and personal communication. The earliest examples of autonomous poetry can be traced back to Oulipo's lo-fi assemblages of text from existing poems. But it is with Manarung, Gervas, and Colton at the turn of the century that systems used fundamentally poetic properties, like rhyme and meter, to move beyond the experimental and arbitrary combination of language $[5,7,11]$. For an expanded review of poetry generation, look to Gonçalo Oliveira 2017 [9].

\section{Joke Generation}

Joke generation has been one of the most fervent areas of NLG within CC. Researchers initially focused on pun generation, where knowledge intensive protocols were used to align contrary antitheses and comparisons (Binsted and Ritchie, 1994; Shah, 2016)[1, 13]. In more recent years researchers have moved on to broader and more sophisticated levels or humoristic language, where structure is less forgiving $[2,13]$. Also falling under joke generation, Oliveira's meme generator exploits the formalities of the medium within a working system.[9]. For a survey of joke generation, look to Section 6 of Gatt and Krahmer's 2017 book chapter, which is also a valuable study of NLG more broadly, and highlights the value in connections between CC and NLG [6].

\section{Conclusion}

CCNLG is a recently initiated workshop within the shifting field of NLG, that serves to expose macro trajectories for creative projects, and discuss their merit on formal and theoretical levels. At a juncture of two fields, CCNLG sits in a challenging yet interesting location, where researchers can take advantage of a broad set of innovative methods and perplexing applications. However, the end tasks - joke, story, or poetry generation hold established bodies of literature and rich histories; it is therefore crucial that we are aware of this work as we look forward.

\section{References}

[1] Kim Binsted and Graeme Ritchie. "An Implemented Model of Punning Riddles". In: Proceedings of the Twelfth National Conference on Artificial Intelligence. AAAI. Seattle, Washington: AAAI Press, 1994, pp. 148-160. ISBN: 978-0-262-51078-3.

[2] Kim Binsted et al. "Computational $\mathrm{Hu}$ mor". In: IEEE Intelligent Systems 21.2 (2006), pp. 59-69. ISSN: 1541-1672. DOI: 10. 1109 / MIS . 2006 . 22. URL: https : / / www . research. ed. ac . uk / portal / en / publications / computational humor(4837fac1 - cffa - 4d55 b456 - 1691a4db2308) /export. html (visited on 09/16/2018).

[3] C. Callaway and J. Lester. "Narrative Prose Generation". In: Articial Intelligence 139.2 (Aug. 2002), pp. 213-252. DOI: https : / / www . sciencedirect . com / science / article / pii / S0004370202002308? via \% 3Dihub. URL: https : / / pdfs . semanticscholar . org / 0013 / 586baf18 ddd9d6b0408f540b808053a76a61. pdf. 
[4] Yun-Gyung Cheong and R. Michael Young. "Narrative Generation for Suspense: Modeling and Evaluation". In: Joint International Conference on Interactive Digital Storytelling. Springer, 2008, pp. 144-155. URL: http: / / link. springer.com/ chapter/10 . 1007/978-3-54089454-4_21 (visited on 01/24/2017).

[5] Simon Colton, Jacob Goodwin, and Tony Veale. "Full-FACE Poetry Generation". In: Proceedings of the Third International Conference on Computational Creativity. 2012, pp. 95-102. URL: http : / / computationalcreativity . net / iccc2012/wp-content / uploads / 2012/05/095-Colton.pdf (visited on $02 / 01 / 2017$ ).

[6] Albert Gatt and Emiel Krahmer. "Survey of the State of the Art in Natural Language Generation: Core Tasks, Applications and Evaluation". In: (Mar. 29, 2017). arXiv: 1703.09902 [CS]. URL: http : / / arxiv.org/abs/1703.09902 (visited on 10/16/2018).

[7] Pablo Gervás. "An Expert System for the Composition of Formal Spanish Poetry". In: Knowledge-Based Systems 14.3-4 (June 2001), pp. 181-188. ISSN: 0950-7051. DOI: $10.1016 /$ S0950-7051 (01)000958. URL: https : / / doi . org/ 10 . $1016 /$ S0950-7051(01) $00095-8$ (visited on 09/11/2018).

[8] Pablo Gervás. "Computational Approaches to Storytelling and Creativity". In: AI Magazine 30.3 (2009), p. 49. URL: http: / / www . aaai . org/ojs/index . php / aimagazine/article/viewFile/ $2250 / 2101$ (visited on 07/13/2017).

[9] Hugo Gonçalo Oliveira. "A Survey on Intelligent Poetry Generation: Languages, Features, Techniques, Reutilisation and Evaluation". In: Proceedings of the 10th International Conference on Natural Language Generation. The 10th International Conference on Natural Language Generation. Santiago de Compostela, Spain: Association for Computational Linguistics, 2017, pp. 11-20. DOI: 10 . 18653 / v1/W173502. URL: http: / / aclweb. org / anthology / W17 - 3502 (visited on 09/11/2018).
[10] M. Lebowitz. "Story Telling as Planning and Learning". In: Columbia University Computer Science Technical Reports, CUCS-202-85 14.1985 (1986), pp. 483502. DoI: https : / / doi . org / 10 . 7916 / D8VX0QJZ. URL: https: / / academiccommons . columbia. edu / doi / 10 . 7916 / D8K362MH / download.

[11] Hisar Maruli Manurung. "An Evolutionary Algorithm Approach to Poetry Generation". Edinburgh: University of Edinburgh. College of Science and Engineering. School of Informatics., July 2004. URL: https : / / www . era . lib.ed . ac.uk/bitstream/handle/1842/ $314 /$ IP 040022 . pdf ? sequence $=1$ \& isAl lowed $=y$.

[12] Neil McIntyre and Mirella Lapata. "Learning to Tell Tales: A Data-Driven Approach to Story Generation". In: Proceedings of the Joint Conference of the 47th Annual Meeting of the ACL and the 4th International Joint Conference on Natural Language Processing of the AFNLP: Volume 1-Volume 1. Association for Computational Linguistics, 2009, pp. 217-225. ISBN: 1-932432-45-0.

[13] Priyanshi R Shah. "Computational Creativity: Automated Pun Generation". In: International Journal of Computer Applications 140 (2016), p. 5. URL: https: / / pdfs . semanticscholar . org / 8255 / d25db977107357f670c132690282164655ed. pdf. 